\title{
Association of serum choline levels and all-cause mortality risk in adults with hypertension: a nested case-control study
}

\author{
Mengmeng Song ${ }^{1,2}$, Benjamin P. Xü ${ }^{3}$, Qiongyue Liang ${ }^{4}$, Yaping Wei ${ }^{5}$, Yun Song ${ }^{6,7}$, Ping Chen ${ }^{8}$, Ziyi Zhou ${ }^{7,9}$,

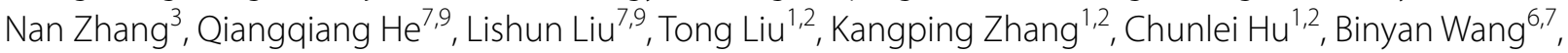 \\ Xiping $\mathrm{Xu}^{5^{*}}$ and Hanping $\mathrm{Shi}^{1,2^{*}}$
}

\begin{abstract}
Background: Serum choline levels were associated with multiple chronic diseases. However, the association between serum choline and all-cause mortality in Chinese adults with hypertension remains unclear. The purpose of this study is to explore the association between serum choline concentrations and all-cause mortality risk in Chinese adults with hypertension, a high-risk population.

Methods: A nested, case-control study was conducted that included 279 patients with all-cause death, and 279 matched, living controls, derived from the China Stroke Primary Prevention Trial (CSPPT). Baseline serum choline concentrations were measured by liquid chromatography with tandem quadrupole mass spectrometry (LC-MS/ MS). Multivariate logistic regression analysis was used to assess the association of serum choline levels and all-cause mortality risk, with adjustment of pertinent covariables, including folic acid and homocysteine.

Results: The median age of all participants was 64.13 years [interquartile range (IQR), 57.33-70.59 years]. The median serum choline concentration for cases $(9.51 \mu \mathrm{g} / \mathrm{mL})$ was higher than that in controls $(7.80 \mu \mathrm{g} / \mathrm{mL})(P=0.009)$. When serum choline concentration was assessed as a continuous variable (per SD increased), there was a positive relation between serum choline levels and all-cause mortality risk [odds ratios (OR), 1.29; 95\% confidence intervals (95\%Cl), $1.06-1.57 ; P=0.010]$. There was an increased all-cause mortality risk for participants in quartiles $2-4(\geq 4.00 \mu \mathrm{g} / \mathrm{mL}$; $\mathrm{OR}, 1.79 ; 95 \% \mathrm{Cl}, 1.15-2.78$ compared with quartile $1(<4.00 \mu \mathrm{g} / \mathrm{mL})$. In addition, non-drinking was found to promote the incidence of all-cause mortality for those with high choline concentrations.
\end{abstract}

Conclusions: High serum choline concentrations were associated with increased all-cause mortality risk among Chinese adults with hypertension, compared to lower choline concentrations.

\footnotetext{
*Correspondence: xipingxu126@126.com; shihp@ccmu.edu.cn

${ }^{1}$ Department of Gastrointestinal Surgery/Clinical Nutrition, Capital Medical University Affiliated Beijing Shijitan Hospital, Beijing 100038, China

${ }^{5}$ Key Laboratory of Precision Nutrition and Food Quality, Ministry of Education, Department of Nutrition and Health, Beijing Advanced Innovation Center for Food Nutrition and Human Health, College of Food Sciences and Nutritional Engineering, China Agricultural University, Beijing 100083, China
}

Full list of author information is available at the end of the article permits use, sharing, adaptation, distribution and reproduction in any medium or format, as long as you give appropriate credit to the original author(s) and the source, provide a link to the Creative Commons licence, and indicate if changes were made. The images or other third party material in this article are included in the article's Creative Commons licence, unless indicated otherwise in a credit line to the material. If material is not included in the article's Creative Commons licence and your intended use is not permitted by statutory regulation or exceeds the permitted use, you will need to obtain permission directly from the copyright holder. To view a copy of this licence, visit http://creativecommons.org/licenses/by/4.0/. The Creative Commons Public Domain Dedication waiver (http://creativeco mmons.org/publicdomain/zero/1.0/) applies to the data made available in this article, unless otherwise stated in a credit line to the data. 
Trial registration clinicaltrials.gov Identifier: NCT007948885; UTL: https://clinicaltrials.gov/ct2/show/NCT00794885? term $=$ NCT00794885\&draw $=2 \&$ rank $=1$.

Keywords: Hypertension, All-cause mortality, Serum choline, Nested case-control

\section{Introduction}

All-cause mortality is one of the basic and most important indicators of a population's health and is readily available from global and regional vital statistics. In an early report based on post hoc analyses of China Stroke Primary Prevention Trial (CSPPT) [1], baseline total homocysteine (tHcy) predicted a 5-year all-cause mortality independent of major lifestyle, and cardiovascular and metabolic risk factors. This study investigates the role of choline, another participant of one carbon metabolism, in all-cause mortality.

Choline is an essential micronutrient with a range of physiological functions in the body [2]. For example, choline serves as a precursor for phospholipids and acetylcholine and has been shown to affect neurodevelopment in rodents [3]. Choline is a major source of methyl donor to betaine [4], which is important for re-methylation of homocysteine to methionine as well as DNA and histone methylation. Choline has been associated with cancer [5], liver steatosis [6], cardiovascular disease and metabolic syndrome [7], however, the findings were inconsistent. Experimental studies reported that choline deficiency was associated with liver disease, atherosclerosis and possibly nervous system diseases [8], whereas choline or betaine supplementation ameliorated liver damage [9]. However, high choline intake was associated with increased cardiometabolic mortality in studies from three racially diverse populations [10]. In addition, studies have reported that high levels of plasma choline are associated with adverse cardiovascular risk factors [11]. Choline consumption may be protective for cancer [12], however, higher serum choline concentration was associated with increased risk of cancer [13]. Therefore, available data indicate that choline could be beneficial or harmful, depending on its levels and other covariables. It underscores the importance to identify optimal range of choline levels to maximize its health benefits and minimize undue harm.

Currently, epidemiologic evidence on the associations of choline with mortality remains limited and inconsistent. Two large US cohort studies revealed positive associations of choline intake with all-cause and CVD mortality [14], but a prospective study of Japanese adults showed no such associations [15]. Reversely, a large prospective cohort study revealed that higher serum choline levels at diagnosis were associated with better hepatocellular carcinoma survival outcomes [16]. Hence, in this large, prospective, follow-up study of the CSPPT, we performed a nested case-control study to assess the prospective association between the baseline serum choline concentrations and all-cause mortality risk in adults with hypertension, and further evaluated whether the associations were modified by sociodemographic and clinical characteristics and lifestyle factors.

\section{Material and methods \\ Participants}

The participants for this nested, case-control study were obtained from the China Stroke Primary Prevention Trial (CSPPT). The methods and major results of the CSPPT have been reported elsewhere [17]. In brief, the CSPPT was a multi-community, randomized, double-blind, controlled trial conducted from May 19, 2008 to August 24, 2013 in 32 communities in China. Eligible participants included men and women aged 45-75 years with hypertension. Hypertension was defined as: (1) seated, resting, systolic blood pressure (SBP) $\geq 140 \mathrm{mmHg}$; or (2) diastolic blood pressure (DBP) $\geq 90 \mathrm{mmHg}$; or (3) taking antihypertensive medication. The major exclusion criteria included history of physician-diagnosed stroke, myocardial infarction, heart failure, post-coronary revascularization, and/or congenital heart disease.

The CSPPT was approved by the Ethics Committee of the Institute of Biomedicine, Anhui Medical University, Hefei, China (FWA assurance number: FWA00001263) and registered with Clinical Trials.gov, NCT00794885. Written, informed consent was obtained from all participants prior to data collection.

\section{Intervention and endpoint events}

In the CSPPT, a total of 20,702 eligible participants were randomly allocated, in a 1:1 ratio, to one of two different treatment groups: a daily oral dose of one tablet containing $10 \mathrm{mg}$ enalapril only (the enalapril group); or a daily oral dose of one tablet containing $10 \mathrm{mg}$ enalapril and $0.8 \mathrm{mg}$ folic acid (the enalapril-folic acid group). All participants were followed up every 3 months where information on vital signs, study drug adherence, concomitant medication use, adverse events and possible endpoint events was collected and recorded by trained research staff and physicians.

Death events, a pre-specified endpoint of the CSPPT, was one of the second outcomes in this study. All cause death included death from any cause (cancer, stroke, 
etc.). Evidence of death was identified by hospital death certificates or home visit reports from investigators. All endpoint outcomes were assessed by the Endpoint Adjudication Committee of the study.

\section{Nested case-control study}

During a median treatment duration of 4.5 years (interquartile range, IQR: $4.2-4.7$ years), a total of 622 participants died. A nested, case-control study was established that was derived from the 622 deceased patients who were matched with 622 living controls within this cohort. Controls were selected from those remaining participants who were still alive, and were matched by age ( \pm 1 year), sex, treatment group (Enalapril and Enalapril-folic acid treatment) and study site with the cases in a 1:1 ratio. Although levels of serum choline vary according to the seasons of sampling, the significant difference can't be explained by seasons only. Thus, we selected the participants from Lianyungang center (the majority of the population) in this current study. For the current analysis, 279 cases and 279 matched controls were included after excluding those with missing data and unpaired individuals (Fig. 1).

\section{Laboratory assays}

At the baseline screening, morning serum samples were collected from all patients following an overnight fast. C677T gene (rs1801133) polymorphisms of 5,10-methylenetetrahydrofolate reductase (MTHFR), one of the genetic determinators of plasma homocysteine (HCY) levels, were detected with an ABI Prism 7900HT sequence detection system (Life Technologies) using the TaqMan assay. Serum HCY and creatinine were both measured using the cycling enzymatic method. Fasting blood glucose (FBG) was analyzed with the hexokinase/ glucose-6-phosphate dehydrogenase method. Triglycerides (TG) and total cholesterol (TC) were both measured using the enzymatic colorimetric method. Serum high-density lipoprotein cholesterol (HDL-C) was measured using the direct test method, serum uric acid (SUA) was measured with the oxidase method, and serum folic acid was measured using a chemiluminescent immunoassay (New Industrial). Biochemical indexes were analyzed using automatic clinical analyzers (Beckman Coulter) at the central laboratory of the National Clinical Research Center for Kidney Disease, (Nanfang Hospital, Guangzhou, China). Plasma choline concentrations were measured by stable-isotope-dilution liquid chromatography-tandem mass spectrometry using 4500MD (AB

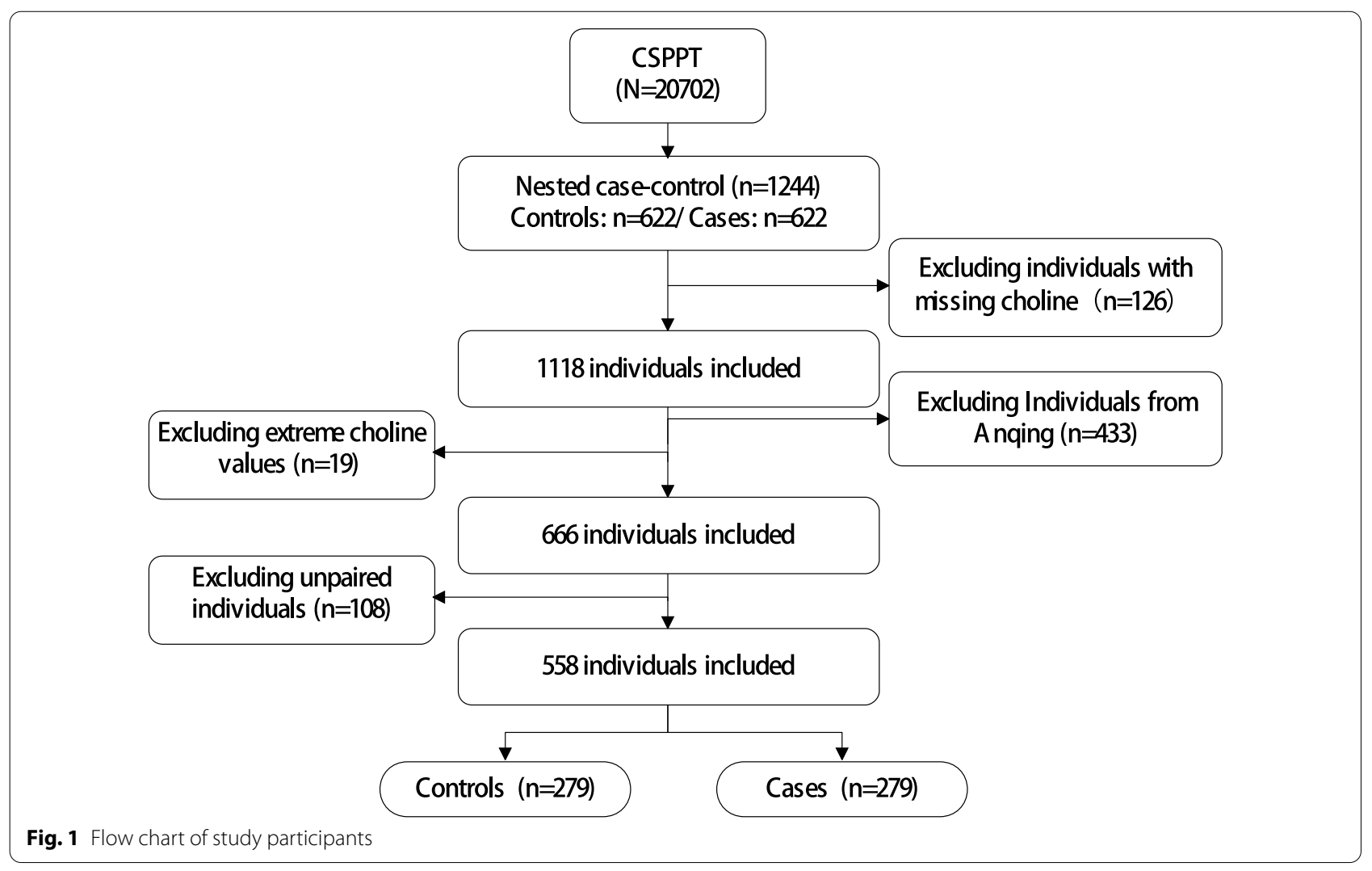


SCIEX) in a commercial lab (Shenzhen Tailored Medical Laboratory, China).

\section{Statistical analysis}

Continuous data were expressed as a median with interquartile range, and categorical data were expressed as $\mathrm{n}(\%)$. Wilcoxon signed rank test were used to compare central estimates across ordered groups for non-normally distributed variables. Chi-square tests were used to compare proportions between groups.

Odds ratios (ORs) of all-cause mortality in relation to serum concentrations of choline were calculated using multivariate logistic regression models. Serum choline concentrations were categorized into quartiles based on its distribution. Multivariate logistic regression models were adjusted for age, body mass index (BMI), sex, month of blood sample collection, treatment group, smoking status, alcohol drinking, MTHFR C677T, systolic blood pressure, and triglyceride, cholesterol, folic acid, uric acid, glucose and homocysteine levels. In the stratified analysis, possible modifications of the association between serum choline as a categorical variable (high vs. low) and the all-cause mortality risks were assessed for variables including age $(<65, \geq 65$ years), sex, BMI $\left(<24.0 \mathrm{k} / \mathrm{m}^{2}, \geq 24.0 \mathrm{~kg} / \mathrm{m}^{2}\right)$, treatment group, smoking (yes, no), alcohol drinking (yes, no), MTHFR genotype $(\mathrm{CC}+\mathrm{CT}$, TT), homocysteine $(<13.72 \mu \mathrm{mol} / \mathrm{L}, \geq 13.72$ $\mu \mathrm{mol} / \mathrm{L})$, glucose $(<5.63 \mathrm{mmol} / \mathrm{L}, \geq 5.63 \mathrm{mmol} / \mathrm{L})$, total cholesterol $(<5.54 \mathrm{mmol} / \mathrm{L}, \geq 5.54 \mathrm{mmol} / \mathrm{L})$ and folic acid $(<6.498 \mathrm{ng} / \mathrm{mL}, \geq 6.498 \mathrm{ng} / \mathrm{mL})$ levels at baseline, using the median for cutoff points.

A two-tailed $P<0.05$ was considered statistically significant in all analyses. $\mathrm{R}$ software (Version 4.0.4, http:// www.R-project.org/) was used for all statistical analyses.

\section{Results}

\section{Characteristics of the participants}

This study included 279 cases of all-cause mortality during follow-up, and 279 matched living controls from the CSPPT cohort (Fig. 1). Among the 279 cases, 39 (14.0\%) died of cancer and $10(3.4 \%)$ from stroke. The median age of all participates was 64.13 years (IQR, 57.3370.59 years). The median serum choline concentration was $8.99 \mu \mathrm{g} / \mathrm{mL}$ (IQR, $4.00-13.33 \mu \mathrm{g} / \mathrm{mL}$ ). There were no major differences in baseline characteristics between cases and controls, other than cases had significantly higher choline concentrations $(9.51 \mu \mathrm{g} / \mathrm{mL}$ vs. $7.80 \mu \mathrm{g} /$ $\mathrm{mL}, P=0.009$ ) (Table 1).

\section{Association of serum choline concentration with the all-cause mortality risk}

The restricted cubic spline graph shows a positive association between serum choline concentration and all-cause mortality risk (Fig. 2). Overall, the all-cause mortality risk increased with the increase of serum choline concentration in hypertensive adults.

Consistently, when serum choline concentration was assessed as a continuous variable, a risk of mortality was found (OR, 1.29; 95\%CI: 1.06-1.57, $P=0.010$ ) with each SD increase of choline (per SD increase). When serum choline concentration was classified as quartiles, a significantly higher risk of all-cause mortality was found in participants in quartile $4(\geq 13.33 \mu \mathrm{g} / \mathrm{mL}$, OR, 2.40; $95 \%$ CI: $1.37-4.20 ; P=0.002)$ compared with participants in quartile $1(<4.00 \mu \mathrm{g} / \mathrm{mL})$. When quartiles 2 to 4 were combined together, a significant increased risk of allcause mortality was found when compared to quartile 1 (OR, 1.79; 95\%CI, 1.15-2.78, $P=0.010$ ) (Table 2).

\section{Stratified analyses by potential effect modifiers}

Stratified analyses were performed to assess the association between serum choline concentrations and all-cause mortality risk in various subgroups (Fig. 3).

For those with increased choline concentrations, an increased risk of all-cause mortality was associated with female, treatment with enalapril, low BMI $\left(<24 \mathrm{~kg} / \mathrm{m}^{2}\right)$, enalapril treatment group, no drinking, no smoking, high glucose $(\geq 5.63 \mathrm{mmol} / \mathrm{L})$ and high total cholesterol $(\geq 5.54 \mathrm{mmol} / \mathrm{L})$. In addition, no-drinking were found to increase the all-cause mortality risk of patients with high serum choline concentrations, along with a significant interaction effect ( $P$ for interaction $=0.017$ ). The visual interaction effect of drinking and serum choline on the all-cause mortality risk was shown in Fig. 4.

\section{Discussion}

In this nested, case-control study which was derived from a prospective follow-up of the CSPPT, we found that among adults with hypertension, high serum choline concentrations $(\geq 4.00 \mu \mathrm{g} / \mathrm{mL})$ were associated with an increased risk of all-cause mortality compared to those with low serum choline levels $(<4.00 \mu \mathrm{g} / \mathrm{mL}$, quartile $1)$. High levels $(\geq 4.00 \mu \mathrm{g} / \mathrm{mL})$ of choline in combination with being female, enalapril treatment, low BMI, neverdrinking, never-smoking, high glucose or high total cholesterol were associated with a higher risk of all-cause mortality in adults with hypertension, and a significant interaction effect was found in those who were non-alcohol drinkers with a high serum choline concentration.

Choline is an essential nutrient for the normal functioning of cell membranes and muscle function, cholinergic neurotransmission, lipid transport and one-carbon metabolism [18]. Imbalanced choline intake can lead to fatty liver development, muscle damage [8], cardiovascular disease [19], cancer [20], cognitive decline [21] and osteoporosis [22]. In addition, the association between 
Table 1 Baseline characteristics of cases and control

\begin{tabular}{|c|c|c|c|c|}
\hline Variables & All patients $(n=558)$ & Controls $(n=279)$ & Cases $(n=279)$ & $P$ \\
\hline Age, years & $64.13(57.33-70.59)$ & $64.13(57.33-70.57)$ & $64.12(57.34-70.72)$ & 0.976 \\
\hline Male, n (\%) & $318(57.0)$ & $159(57.0)$ & $159(57.0)$ & 1.000 \\
\hline $\mathrm{BMI}, \mathrm{kg} / \mathrm{m}^{2}$ & $24.54(22.24-27.18)$ & 25.04 (22.64-27.34) & 24.34 (21.84-26.73) & 0.033 \\
\hline Current smoking, n (\%) & $190(34.1)$ & $93(33.3)$ & $97(34.8)$ & 0.925 \\
\hline Current drinking, n (\%) & $181(32.4)$ & $96(34.4)$ & $85(30.5)$ & 0.551 \\
\hline Baseline SBP, mmHg & $168.00(156.00-181.83)$ & $165.33(154.67-180.67)$ & $170.67(157.33-183.00)$ & 0.081 \\
\hline Baseline DBP, mmHg & 94.67 (88.00-101.33) & $93.33(88.0-100.0)$ & 96.67 (88.00-102.00) & 0.152 \\
\hline \multicolumn{5}{|l|}{ Treatment group } \\
\hline Enalapril & $298(53.4)$ & $149(53.4)$ & $149(53.4)$ & \multirow[t]{2}{*}{1.000} \\
\hline Enalapril-folic acid & $260(46.6)$ & $130(46.6)$ & $130(46.6)$ & \\
\hline \multicolumn{5}{|l|}{ MTHFR C677T } \\
\hline $\mathrm{CC}$ & $137(24.6)$ & $70(25.1)$ & $67(24.0)$ & \multirow[t]{3}{*}{0.626} \\
\hline $\mathrm{CT}$ & $277(49.6)$ & $142(50.9)$ & $135(48.4)$ & \\
\hline $\mathrm{TT}$ & $144(25.8)$ & $67(24.0)$ & $77(27.6)$ & \\
\hline Triglyceride, mmol/L & $1.43(1.06-1.99)$ & $1.47(1.05-2.09)$ & $1.38(1.07-1.93)$ & 0.295 \\
\hline Total cholesterol, mmol/L & $5.54(4.78-6.22)$ & $5.54(4.94-6.21)$ & $5.54(4.64-6.22)$ & 0.407 \\
\hline Uric acid, mg/dL & $304.00(256.00-356.00)$ & $302.00(254.50-354.00)$ & $305.00(257.00-361.00)$ & 0.437 \\
\hline $\mathrm{HDL}$ cholesterol, mmol/L & $1.29(1.07-1.52)$ & $1.29(1.09-1.52)$ & $1.29(1.05-1.58)$ & 0.967 \\
\hline Glucose, $\mathrm{mmol} / \mathrm{L}$ & $5.63(5.17-6.34)$ & $5.58(5.17-6.32)$ & $5.63(5.19-6.40)$ & 0.411 \\
\hline Creatinine, $\mathrm{mmol} / \mathrm{L}$ & $68.70(58.30-79.28)$ & $68.70(57.35-80.05)$ & $68.70(58.55-76.95)$ & 0.703 \\
\hline Serum Folic acid, ng/mL & $6.50(4.87-9.09)$ & $6.57(4.66-9.20)$ & $6.50(4.94-8.82)$ & 0.915 \\
\hline Homocysteine, $\mu \mathrm{mol} / \mathrm{L}$ & $13.72(11.02-18.82)$ & $13.70(10.78-18.27)$ & $13.72(11.17-19.25)$ & 0.289 \\
\hline Choline, $\mu \mathrm{g} / \mathrm{ml}$ & $8.99(4.00-13.33)$ & $7.80(3.37-12.72)$ & $9.51(4.45-13.89)$ & 0.009 \\
\hline
\end{tabular}

Continuous variables are presented as median (quantile1-quantile3), categorical variables are presented as $\mathrm{n}(\%)$.

Differences in baseline characteristics between cases and controls were compared using $\mathrm{x}^{2}$ tests for categorical variables and Wilcoxon signed rank tests for continuous variables.

SD, standard deviation; SBP, systolic blood pressure; DBP, diastolic blood pressure; MTHFR, 5,10-methylenetetrahydrofolate reductase; CC, CT and TT are different genotypes of MTHFR.

high choline intake/plasma choline level and mortality risk is controversial [15, 23, 24]. However, most previous studies focused on choline intake and cardiovascular disease mortality risk rather than serum choline and all-cause mortality. In addition, a meta-analysis showed that elevated concentrations of choline were associated with an increased risk of major adverse cardiac events (MACE, including death, myocardial infraction, stroke) independently of traditional risk factors [24]. However, the main outcomes of this meta-analysis included events other than death. Our study is the first to focus on the association of serum choline concentration and all-cause mortality in participants with hypertension, and found that high serum choline was associated with increased risk of all-cause mortality.

Cancer and cardiovascular disease (CVD) represent the leading cause of death and morbidity worldwide. Among the cases in our study, $14.0 \%$ and $3.4 \%$ developed cancer and stroke, respectively, during follow-up. A history of diabetes or CVD strengthened the choline-mortality association, as shown in many previous studies $[14,25$,
26]. The participants in our study had hypertension, a risk factor of CVD, which may have strengthened the association between choline and mortality. In addition, those participants with both high serum choline and high total cholesterol had a higher risk of mortality, a finding that is consistent with previous studies, where it has been shown that clinical-based risks of CVD increased the choline-mortality association [27]. Another study showed that higher concentrations of plasma choline were associated with an unfavorable cardiometabolic risk-factor profile, including higher body mass index (BMI) [32]. Our study did not find the significant difference of BMI between participant with different serum choline level, probably because this study population was the adults with hypertension, with an overall high BMI.

Interestingly, we observed a trend of an inverse association of choline intake with all-cause mortality among alcohol drinkers or smokers. Given that choline is an important factor in maintaining liver function and that the vast majority of choline metabolism occurs in the liver [28], it is probable that in times of choline 


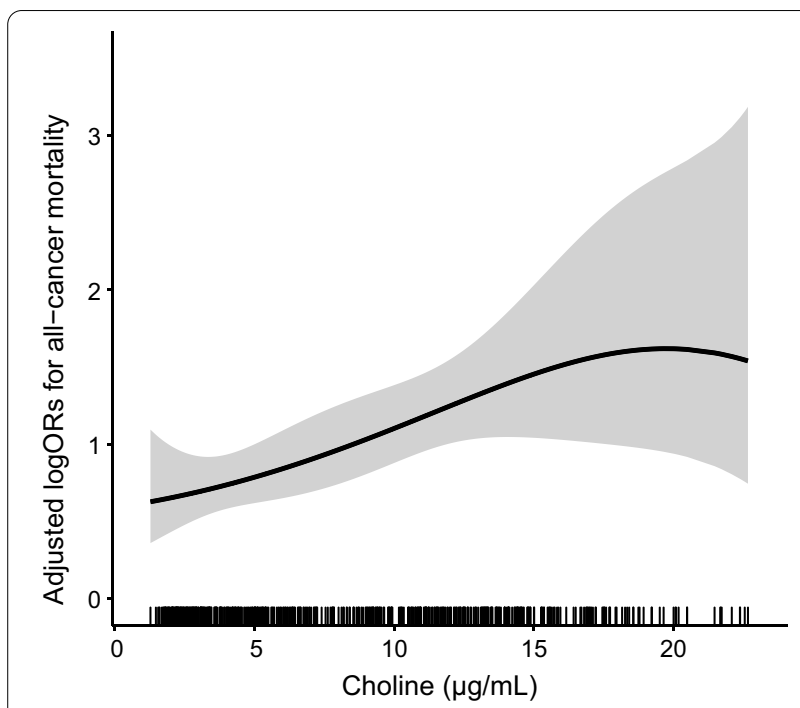

Fig. 2 The association between baseline serum choline and all-cause mortality risk of participants. The splines were adjusted for body mass index, month of collecting blood samples, smoking, alcohol drinking, MTHFR C677T, systolic blood pressure, triglyceride, cholesterol, uric acid, folic acid, baseline fasting blood glucose, homocysteine and the matching factors (age, sex, treatment group)

deprivation, varying degrees of liver damage and liver diseases may develop [29, 30]. As we all known, alcohol consumption was a main factor of liver function damage. High serum choline levels reflect high levels of choline metabolism, and a high choline level may help recover liver damage from excessive alcohol drinking and, in turn, lead to a phenomenon of reduced all-cause mortality risk happened in alcohol drinkers with high serum choline level. We had no relative data on liver function biomarkers in our study, but we observed an obvious inverse association of serum choline with deaths from all-cause among drinkers. In future studies investigating the health effects of choline-related nutrients, it will be important to take a patient's cardiometabolic disease status and alcohol drinking status into consideration. In addition, a previous study reported that smoking decreases serum choline levels [31], which may be the cause the inverse phenomenon observed in our study.

However, it seems that the serum choline concentrations in our study are higher than those reported in previous studies $[32,33]$. We explored four possibilities that may affect serum choline concentrations: laboratory measurement, study population of hypertension, the crosstalk of choline and folic acid., and sample storage length. Firstly, we worked with the lab that measured choline for this study to examine the purity of standard, detailed monitoring parameters, linearity curve, and reproducibility, and all of which met the quality control standards, indicating that the choline measurements for this study were accurate and reproducible. The second possibility is related to the study population with hypertension. This study was conducted in a hypertensive

Table 2 The association between baseline serum choline and all-cause mortality risk

\begin{tabular}{|c|c|c|c|c|c|}
\hline \multirow[t]{2}{*}{ Choline, $\mu \mathrm{g} / \mathrm{mL}$} & \multirow[t]{2}{*}{ Cases/controls } & \multicolumn{2}{|l|}{ Crude model } & \multicolumn{2}{|l|}{ Adjusted model } \\
\hline & & OR $(95 \% \mathrm{Cl})$ & $P$ & OR $(95 \% \mathrm{Cl})$ & $P$ \\
\hline Per SD increased & $279 / 279$ & $1.20(1.02,1.41)$ & 0.026 & $1.29(1.06-1.57)$ & 0.010 \\
\hline \multicolumn{6}{|l|}{ Quartiles } \\
\hline Quartile1 (<4.00) & $58 / 82$ & Reference & & Reference & \\
\hline Quartile2 (4.00-8.99) & $71 / 68$ & $1.48(0.92-2.37)$ & 0.107 & $1.59(0.94-2.70)$ & 0.084 \\
\hline Quartile3 (8.99-13.33) & $70 / 69$ & $1.43(0.89-2.30)$ & 0.135 & $1.59(0.92-2.73)$ & 0.094 \\
\hline Quartile4 ( $\geq 13.33$ ) & $80 / 60$ & $1.89(1.17-3.03)$ & 0.009 & $2.40(1.37-4.20)$ & 0.002 \\
\hline$P$ for trend & & & 0.010 & & 0.005 \\
\hline
\end{tabular}

Categories

$\begin{array}{lllll}\text { Quartile1 }(<4.00) & 58 / 82 & \text { Reference } & & \text { Reference } \\ \text { Quartile2-Quartile4 }(\geq 4.00) & 221 / 197 & 1.59(1.08-2.34) & 0.020 & 1.79(1.15-2.78\end{array}$

Quartile2-Quartile4 ( $\geq 4.00) \quad 221 / 197 \quad 1.59(1.08-2.34) \quad 0.020 \quad 1.79(1.15-2.78)$

Adjusted for age, body mass index, sex, month of collecting blood samples, treatment group, smoking status, alcohol drinking, MTHFR C677T, systolic blood pressure, triglyceride, cholesterol, folic acid, uric acid, glucose, homocysteine

(See figure on next page.)

Fig. 3 The association between serum choline concentrations and all-cause mortality risk in various subgroups. The association between serum choline and all-cause mortality risk in various subgroups in the nested case-control study within the China Stroke Primary Prevention Trial, conducted between May 2008 and August 2013. Adjusted for age, BMI, sex, treatment group, smoking status, alcohol drinking, MTHFR C677T, systolic blood pressure, triglyceride, cholesterol, uric acid, glucose, homocysteine, folic acid levels and month of collecting blood samples, if not stratified. OR, odds ratio. 95\%Cl, 95\% confidence interval 


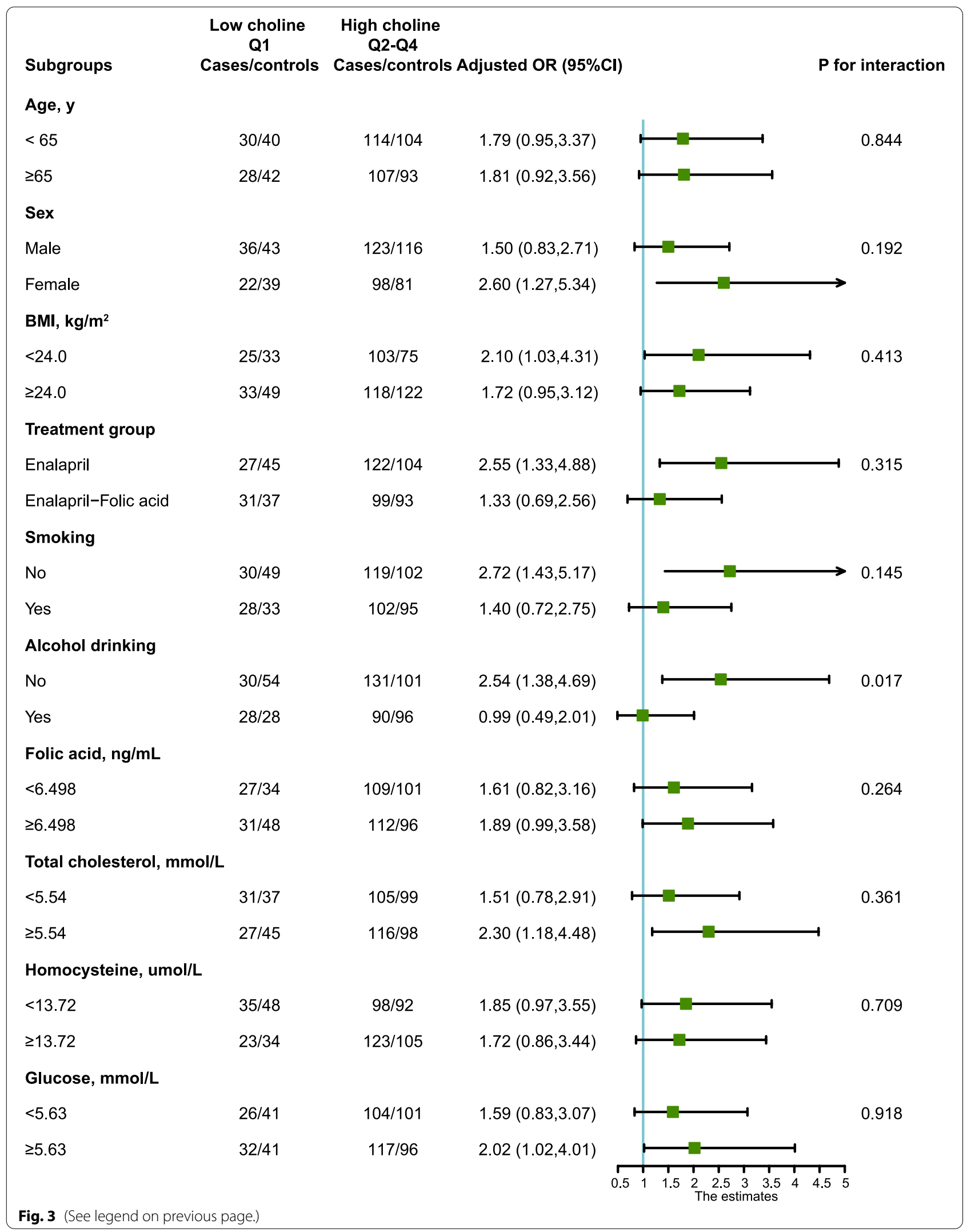




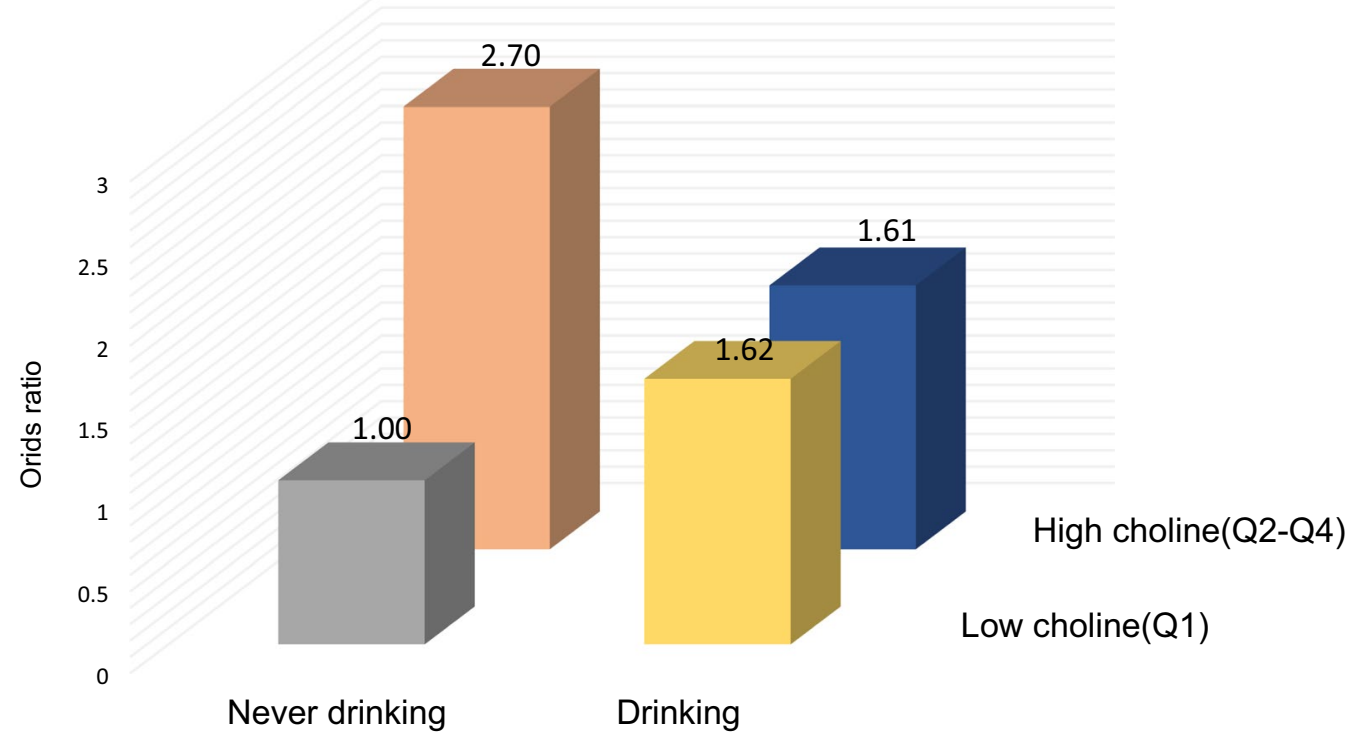

Fig. 4 Interaction effect associations of baseline serum choline and alcohol with all-cause mortality risk

population with a mean age of 64 years. A recent choline study in Chinese population with a mean age of 58 years reported higher choline level in hypertensive participants compared to non-hypertensive controls [34]. Thus, our observed higher choline levels could be due to the fact that all of the study participants in our study had hypertension. This possible link between choline levels and hypertension remains to be verified by additional studies. The third possibility is related to low folate status in the study population. Choline participates in one carbon metabolic cycle, including sharing methylation pathways with folate. One study showed that choline and folate is metabolically inter-related; and choline is utilized as a methyl donor when folate intake is low [35]. As we published previously (17), the baseline serum folate level in the parent study population (CSPPT) is much lower than Western populations such as US population, due to the fact that China has not implemented mandatory folic acid grain fortification and folate intake in typical Chinese diet is low. In our study sample, baseline folic acid level was inversely correlated with choline levels. The complex interaction of choline and folate in patients with hypertension and the underlying mechanism is unclear. We hypothesize that higher serum level of choline may be a marker of low folate status, which is common in the Chinese patients with hypertension. The human body may attempt to absorb more choline from diets to compensate for the low folate status. This hypothesis needs to be explored and verified in the future study. The fourth possibility is related to samples storage and thaw cycle. Our study's serum samples were stored in $-80^{\circ} \mathrm{C}$ freezer for about 10 years and underwent several freezing-thawing cycles. It is unclear to what extent the long-term storage and freezing-thawing cycles could impact choline levels. This again raises questions for future studies to consider, when using long-term stored serum samples, or when comparing findings across studies.

Our current study is novel for it uncovers the association of serum choline levels, a component of one-carbon metabolism, and the risk of all-cause mortality in an adult population with hypertension. The study results indicate that attention should be given to monitoring serum choline concentrations of patients with hypertension. Additionally, its strengths lie in the fact that this nested, case-control study was derived from a large, prospective cohort study, thereby avoiding recall bias. Participant serum choline concentrations were obtained from baseline blood samples prior to death, eliminating inverse causality.

There were also several limitations to this study. First, only baseline serum choline levels of the study participants were obtained. Repeated measurements of serum choline would have provided more information about allcause mortality risk in relation to change in or cumulative serum choline levels. Second, this study was conducted in adults with hypertension, a high-risk population for cardiovascular morbidity and mortality; the extrapolation of these findings to populations without hypertension remains to be determined. However, adjustments for blood pressure measurements at baseline did not substantially change the findings. Third, this study represents a preliminary exploration of the association between 
choline and all-cause mortality risk. Our findings warrant further investigation, including simultaneously considering other components of one carbon metabolism.

\section{Conclusion}

In this adult population with hypertension, we conducted the first prospective evaluation on the independent effect of serum choline concentrations on the risk of all-cause mortality. High serum choline levels were associated with an increased risk of all-cause mortality. These results are of potential scientific and clinical significance for death monitoring and control in adults with hypertension. Further studies are needed to confirm these findings in other populations.

\begin{abstract}
Abbreviations
CSPPT: China stroke primary prevention trial; LC-MS/MS: Liquid chromatography with tandem quadrupole mass spectrometry; IQR: Interquartile range; $O R$ : Odds ratios; 95\%Cl: 95\% Confidence intervals; total tHcy: Homocysteine; SBP: Systolic blood pressure; DBP: Diastolic blood pressure; MTHFR: Polymorphisms of 5,10-methylenetetrahydrofolate reductase; TG: Triglycerides; TC: Total cholesterol; HDL-C: High-density lipoprotein cholesterol; BMI: Body Mass Index; CVD: Cardiovascular disease.
\end{abstract}

\section{Acknowledgements}

We thank all the staff and participants of the CSPPT for their important contributions.

\section{Authors' contributions}

MS: methodology, software, writing- original draft preparation, writingreviewing and editing; BPX: writing- reviewing and editing; QL: methodology, software; YW: writing- reviewing and editing; YS: supervision, validation; PC: supervision, validation; ZZ: supervision, validation; NZ: supervision, validation; QH: supervision, validation; LL: supervision, validation; TL: writing- reviewing and editing; KZ: investigation. CH: investigation; BW supervision, validation; XX: conceptualization, supervision, resources; HS: conceptualization, supervision, validation, writing- reviewing and editing. All authors read and approved the final manuscript.

\section{Funding}

The study was supported by the National Key Research and Development Program [2016YFE0205400, 2018ZX09739010, 2018ZX09301034003]; the Department of Science and Technology of Guangdong Province [2020B121202010]; the Science and Technology Planning Project of Guangzhou, China [201707020010]; the Science, Technology and Innovation Committee of Shenzhen [GJHS20170314114526143, JSGG20180703155802047]; the Economic, Trade and Information Commission of Shenzhen Municipality [20170505161556110, 20,170,505,160,926,390, 201705051617070].

\section{Availability of data and materials}

Data described in the manuscript, code book, and analytic code will be made available upon request after the review and approval of the institutional review board.

\section{Declarations}

\section{Ethics approval and consent to participate}

The CSPPT and the present study were approved by the Ethics Committee of the Institute of Biomedicine, Anhui Medical University, Hefei, China (FWA assurance number: FWA00001263) and is registered with ClinicalTrials.gov, NCT00794885. Written, informed consent was obtained from all participants or their representative relatives.
Consent for publication

Not applicable.

\section{Competing interests}

The authors declare no competing interests.

\section{Author details}

'Department of Gastrointestinal Surgery/Clinical Nutrition, Capital Medical University Affiliated Beijing Shijitan Hospital, Beijing 100038, China. ${ }^{2}$ Beijing International Science and Technology Cooperation Base for Cancer Metabolism and Nutrition, Beijing 100038, China. ${ }^{3}$ Department of Epidemiology and Department of Nutrition, Harvard TH Chan School of Public Health, Boston, MA 02115, USA. ${ }^{4}$ State Key Laboratory of Natural Medicines, Research Center of Biostatistics and Computational Pharmacy, China Pharmaceutical University, Nanjing 210009, China. ${ }^{5}$ Key Laboratory of Precision Nutrition and Food Quality, Ministry of Education, Department of Nutrition and Health, Beijing Advanced Innovation Center for Food Nutrition and Human Health, College of Food Sciences and Nutritional Engineering, China Agricultural University, Beijing 100083, China. ${ }^{6}$ Institute for Biomedicine, Anhui Medical University, Hefei, China. ${ }^{7}$ Shenzhen Evergreen Medical Institute, Shenzhen, China. ${ }^{8}$ College of Pharmacy, Jinan University, Guangzhou, China. ${ }^{9}$ Graduate School at Shenzhen, Tsinghua University, Shenzhen, China.

Received: 24 August 2021 Accepted: 8 December 2021

Published online: 20 December 2021

\section{References}

1. Xu B, Kong X, Xu R, Song Y, Liu L, Zhou Z, et al. Homocysteine and allcause mortality in hypertensive adults without pre-existing cardiovascular conditions: Effect modification by MTHFR C677T polymorphism. Medicine. 2017:96(8):e5862.

2. Hollenbeck CB. An introduction to the nutrition and metabolism of choline. Cent Nerv Syst Agents Med Chem. 2012;12(2):100-13.

3. Blusztajn JK, Slack BE, Mellott TJ. Neuroprotective actions of dietary choline. Nutrients. 2017;9(8):815.

4. Bekdash RA. Neuroprotective effects of choline and other methyl donors. Nutrients. 2019;11(12):2995.

5. Johansson M, Van Guelpen B, Vollset SE, Hultdin J, Bergh A, Key T, et al. One-carbon metabolism and prostate cancer risk: prospective investigation of seven circulating B vitamins and metabolites. Cancer Epidemiol Biomark Prev Publ Am Assoc Cancer Res Cosponsored Am Soc Prev Oncol. 2009;18(5):1538-43.

6. Vance DE, Li Z, Jacobs RL. Hepatic phosphatidylethanolamine N-methyltransferase, unexpected roles in animal biochemistry and physiology. J Biol Chem. 2007;282(46):33237-41.

7. Konstantinova SV, Tell GS, Vollset SE, Ulvik A, Drevon CA, Ueland PM. Dietary patterns, food groups, and nutrients as predictors of plasma choline and betaine in middle-aged and elderly men and women. Am J Clin Nutr. 2008;88(6):1663-9.

8. Zeisel SH, da Costa KA. Choline: an essential nutrient for public health. Nutr Rev. 2009;67(11):615-23.

9. Kitagawa E, Ota Y, Hasegawa M, Nakagawa T, Hayakawa T. Accumulation of liver lipids induced by vitamin B(6) deficiency was effectively ameliorated by choline and to a lesser extent, betaine. J Nutr Sci Vitaminol. 2019:65(1):94-101.

10. Yang JJ, Lipworth LP, Shu XO, Blot WJ, Xiang YB, Steinwandel MD, et al. Associations of choline-related nutrients with cardiometabolic and all-cause mortality: results from 3 prospective cohort studies of blacks, whites, and Chinese. Am J Clin Nutr. 2020;1 11 (3):644-56.

11. Ueland PM. Choline and betaine in health and disease. J Inherit Metab Dis. 2011;34(1):3-15.

12. Sun S, Li X, Ren A, Du M, Du H, Shu Y, et al. Choline and betaine consumption lowers cancer risk: a meta-analysis of epidemiologic studies. Sci Rep. 2016;6:35547.

13. Guertin KA, Li XS, Graubard BI, Albanes D, Weinstein SJ, Goedert JJ, et al. Serum trimethylamine $\mathrm{N}$-oxide, carnitine, choline, and betaine in relation to colorectal cancer risk in the alpha tocopherol, beta carotene cancer prevention study. Cancer Epidemiol Biomark Prev Publ Am Assoc Cancer Res Cosponsored Am Soc Prev Oncol. 2017;26(6):945-52. 
14. Zheng Y, Li Y, Rimm EB, Hu FB, Albert CM, Rexrode KM, et al. Dietary phosphatidylcholine and risk of all-cause and cardiovascular-specific mortality among US women and men. Am J Clin Nutr. 2016;104(1):173-80.

15. Nagata C, Wada K, Tamura T, Konishi K, Kawachi T, Tsuji M, et al. Choline and betaine intakes are not associated with cardiovascular disease mortality risk in japanese men and women. J Nutr. 2015;145(8):1787-92.

16. Liu ZY, Yishake D, Fang AP, Zhang DM, Liao GC, Tan XY, et al. Serum choline is associated with hepatocellular carcinoma survival: a prospective cohort study. Nutr Metab. 2020;17:25.

17. Huo Y, Li J, Qin X, Huang Y, Wang X, Gottesman RF, et al. Efficacy of folic acid therapy in primary prevention of stroke among adults with hypertension in China: the CSPPT randomized clinical trial. JAMA. 2015;313(13):1325-35

18. Zeisel SH. Choline: critical role during fetal development and dietary requirements in adults. Annu Rev Nutr. 2006;26:229-50.

19. Millard HR, Musani SK, Dibaba DT, Talegawkar SA, Taylor HA, Tucker KL, et al. Dietary choline and betaine; associations with subclinical markers of cardiovascular disease risk and incidence of CVD, coronary heart disease and stroke: the Jackson Heart Study. Eur J Nutr. 2018;57(1):51-60.

20. Youn J, Cho E, Lee JE. Association of choline and betaine levels with cancer incidence and survival: a meta-analysis. Clin Nutr (Edinb, Scotl). 2019;38(1):100-9.

21. Ylilauri MPT, Voutilainen S, Lönnroos E, Virtanen HEK, Tuomainen TP, Salonen JT, et al. Associations of dietary choline intake with risk of incident dementia and with cognitive performance: the Kuopio Ischaemic Heart Disease Risk Factor Study. Am J Clin Nutr. 2019;110(6):1416-23.

22. Zhang YW, Lu PP, Li YJ, Dai GC, Cao MM, Xie T, et al. Low dietary choline intake is associated with the risk of osteoporosis in elderly individuals: a population-based study. Food Funct. 2021;12:6442.

23. Mazidi M, Katsiki N, Mikhailidis DP, Banach M. Dietary choline is positively related to overall and cause-specific mortality: results from individuals of the National Health and Nutrition Examination Survey and pooling prospective data. Br J Nutr. 2019;122(11):1262-70.

24. Heianza Y, Ma W, Manson JE, Rexrode KM, Qi L. Gut microbiota metabolites and risk of major adverse cardiovascular disease events and death: a systematic review and meta-analysis of prospective studies. J Am Heart Assoc. 2017;6(7):e004947.

25. Wang Z, Klipfell E, Bennett BJ, Koeth R, Levison BS, Dugar B, et al. Gut flora metabolism of phosphatidylcholine promotes cardiovascular disease. Nature. 2011;472(7341):57-63.

26. Tang WH, Wang Z, Levison BS, Koeth RA, Britt EB, Fu X, et al. Intestinal microbial metabolism of phosphatidylcholine and cardiovascular risk. N Engl J Med. 2013;368(17):1575-84.

27. Zeisel SH, Warrier M. Trimethylamine N-oxide, the microbiome, and heart and kidney disease. Annu Rev Nutr. 2017;37:157-81.

28. Mehedint MG, Zeisel SH. Choline's role in maintaining liver function: new evidence for epigenetic mechanisms. Curr Opin Clin Nutr Metab Care. 2013;16(3):339-45.

29. Buchman AL, Ament ME, Sohel M, Dubin M, Jenden DJ, Roch M, et al. Choline deficiency causes reversible hepatic abnormalities in patients receiving parenteral nutrition: proof of a human choline requirement: a placebo-controlled trial. JPEN J Parenter Enteral Nutr. 2001;25(5):260-8.

30. Mato JM, Lu SC. The hepatocarcinogenic effect of methionine and choline deficient diets: an adaptation to the Warburg effect? Alcohol Clin Exp Res. 2011:35(5):811-4.

31. Konstantinova SV, Tell GS, Vollset SE, Nygård O, Bleie $\varnothing$, Ueland PM. Divergent associations of plasma choline and betaine with components of metabolic syndrome in middle age and elderly men and women. J Nutr. 2008;138(5):914-20.

32. Zhong RH, Long JA, Wang F, Chen S, Luo Y, Lu XT, et al. Association between serum choline and betaine concentrations and longitudinal changes of body composition in community-dwelling middle-aged and older Chinese adults. Appl Physiol Nutr Metab. 2020;45(7):737-44.

33. Nilsson AK, Pedersen A, Malmodin D, Lund AM, Hellgren G, Löfqvist C, et al. Serum choline in extremely preterm infants declines with increasing parenteral nutrition. Eur J Nutr. 2021;60(2):1081-9.

34. Guo F, Qiu X, Zhu Y, Tan Z, Li Z, Ouyang D. Circulating choline is associated with coronary artery stenosis in patients with hypertension: a cross-sectional study of Chinese adults. J Clin Hypertens (Greenwich). 2020;22(11):2069-76.
35. Imbard A, Smulders YM, Barto R, Smith DE, Kok RM, Jakobs C, et al. Plasma choline and betaine correlate with serum folate, plasma S-adenosylmethionine and S-adenosyl-homocysteine in healthy volunteers. Clin Chem Lab Med. 2013;51(3):683-92.

\section{Publisher's Note}

Springer Nature remains neutral with regard to jurisdictional claims in published maps and institutional affiliations.
Ready to submit your research? Choose BMC and benefit from:

- fast, convenient online submission

- thorough peer review by experienced researchers in your field

- rapid publication on acceptance

- support for research data, including large and complex data types

- gold Open Access which fosters wider collaboration and increased citations

- maximum visibility for your research: over $100 \mathrm{M}$ website views per year

At BMC, research is always in progress.

Learn more biomedcentral.com/submissions 